\title{
CellCAN: A Unique Enabler of Regenerative Medicine and Cell Therapy in Canada
}

\author{
Denis-Claude Roy, ${ }^{1, *}$ Anne-Marie Alarco, ${ }^{2}$ and Rosario Isasi ${ }^{3}$
}

CellCAN will promote exchanges, cooperation, partnership development, and innovation in regenerative medicine and cell therapy.

-Dr. Roy, CellCAN CEO and Head, Research Centre at Hôpital

Maisonneuve-Rosemont, Montreal, Canada

\section{ABSTRACT}

Regenerative Medicine and Cell Therapy (RMCT) is paving the way for the most innovative and promising medical breakthroughs of the 21st century. Indeed, its curative potential is immense and builds on the already proven benefits of stem cell transplantation. Successful and broad clinical implementation of RMCT, as well as reaping of its full social and economic benefits, is contingent on the resolution of a range of issues. The CellCAN network, a not-for-profit corporation, was created to tackle these challenges, gathering the key forces of the numerous Canadian organizations involved in basic research, assay development, manufacturing, clinical research, clinical trials, legal and ethical regulations, and policies, all working to move RMCT forward. CellCAN creates a national enterprise by bringing together a community of renowned researchers, industries, clinicians, funders and regulators, and aligning it with cell-handling facilities involved in processing cell products and other products for cell therapy clinical trials to ensure capacity and know-how for stem cell research and efficient execution of cell therapy clinical trials. CellCAN is uniquely positioned to accelerate the implementation of RMCT in Canada and disseminate novel developments and findings, thus significantly contributing to the world's knowledge in cellular therapeutics. As such, the CellCAN model could also serve as a useful benchmark to accelerate RMCT implementation in other countries.

${ }^{1}$ Division of Hematology-Oncology/Stem Cell Transplantation, Hôpital MaisonneuveRosemont Research Center, and Department of Medicine, University of Montreal, Montreal, Quebec, Canada.

2 CellCAN Regenerative Medicine and Cell Therapy Network, Montreal, Quebec, Canada.

${ }^{3}$ Department of Human Genetics, Faculty of Medicine, Centre of Genomics and Policy, McGill University, Montreal, Quebec, Canada.

*(Correspondence: denis-claude.roy@umontreal .ca)

\section{Transforming medical practice in Canada: from treatment to cure}

Transformative innovations such as regenerative medicine and cell therapy (RMCT) will shape the practice of medicine in this 21 st century. Rather than alleviating symptoms, it has the potential

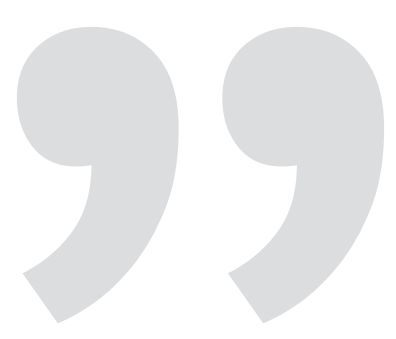

Canada has the capacity to conduct 50-75 new phase I/II cell-based clinical trials over the next 5 years. to cure diseases. RMCT offers hope for diseases for which there is currently no effective cure, as well as for substantial reductions in both the direct and indirect healthcare costs, particularly for chronic diseases such as diabetes and brain degenerative diseases such as Alzheimer, cancer, and cardiac conditions.

It is estimated that in the United States, the current cost of treating all heart diseases is 
about $\$ 273$ billion and over the next 20 years, this cost will reach $\$ 818$ billion [1]. In the United States alone, the direct cost savings of treating chronic diseases such as Parkinson's disease, spinal cord injury, heart failure, stroke, and diabetes with cellular approaches are predicted to be in the order of $\$ 250$ billion. Thus, these diseases are excellent targets for regenerative medicine (RM) [2]. By 2010 more than 300,000 patients had already been treated with RM products [3]. Further, this field of research could create thousands of jobs and economic advancement where RM companies flourish [4-6].

It is therefore no surprise that RMCT remains a priority research area for Canada's government. As recently as September 2011, Prime Minister Harper highlighted this potential in a joint statement with UK Prime Minister Cameron:

Exploiting the fact that Canada and the UK are global leaders in ... regenerative medicine and cell therapy, we will . . foster collaboration, facilitating the translation of our advanced knowledge into life-changing therapies that will benefit our patients, healthcare institutions and industries.

Canada is uniquely placed as one of the earliest beneficiaries of stem cell research. Beginning with the discovery of stem cells by Drs. Till and McCulloch at the Ontario Cancer Institute in 1961, a unique confluence of history and strategic investments has allowed Canada to play a leading role in this "medicine of the future." The establishment of the Stem Cell Network (SCN) as a Network of Centres of Excellence (NCE) in 2001 coalesced Canada's internationally recognized strength in stem cell biology with world-class expertise in bioengineering, pioneering clinical practice, and commercial capabilities with industry partners. Indeed, the SCN has been promoting the development of basic and translational stem cell research, and acting as a catalyst to enable the translation of stem cell discoveries into clinical applications, commercial products, and public policy. Its efforts to link researchers of many disciplines from all across
Canada have been fruitful and particularly efficient at establishing highly productive national multidisciplinary working groups. This has greatly strengthened the international positioning of Canadian stem cell researchers.

In recent years, close to CAD $\$ 100$ million of federal, provincial, and philanthropic funds has been invested in building new and highly specialized good manufacturing practice (GMP) cell and tissue manufacturing facilities across the country, providing Canada with close to 30 new Health Canada-compliant clean rooms and the capacity to conduct 50-75 new phase I/II cell-based clinical trials over the following 5 years.

In addition, the Centre for Commercialization of Regenerative Medicine (CCRM) was established in 2012 to help translate promising discoveries into commercially viable RM products. Supported for five years by the NCE, CCRM is hosted by the University of Toronto.

\section{CellCAN: gathering the driving forces of RMCT} in Canada

Successful and broad clinical implementation of RMCT as well as reaping of its full social and economic benefits are contingent on the resolution of a range of issues, among which is the inaccessibility to available information about RMCT. For example, basic researchers are often unaware of the steps required to take a research innovation to the clinic. The ability to link with a cell therapy center with the required translational expertise would both facilitate and accelerate the development and implementation of novel RMCT applications. In addition, enhanced access to clinically useful protocols and infrastructures, practice guidelines, educational initiatives for key stakeholders, and well-defined requirements and practices for proving clinical utility and implementing new tools would represent a major step forward. Furthermore, as the field of RMCT grows, several regulatory and ethical issues that need to be addressed before RMCT becomes routine medical practice will need to be resolved. It is also crucial to make the lay public aware of the RMCT opportunities without creating unrealistic expectations.

Around the world, regulatory agencies are also developing policy frameworks for safe and effective cell therapies. GMP plays a large role in the regulatory requirements that RMCT product developers must follow. Moreover, cell manufacturing has become a key factor for successful clinical translation. Several studies have noted the existence of a direct relationship between regulation and innovation in the clinical translation and commercialization of scientific discoveries and how regulatory frameworks could enable or hinder innovation [7].

To tackle these challenges, the CellCAN network was created in 2009 thanks to the strong leadership and structuring impetus of the SCN. CellCAN gathered the key forces of the numerous Canadian organizations involved in basic research, assay development, manufacturing, clinical research, clinical trials, legal and ethical regulations, policies, all working to move forward RMCT. To further structure its work, CellCAN was incorporated as a not-for-profit organization in February 2014, hosted at Hôpital MaisonneuveRosemont in Montreal.

CellCAN regroups the main five cell and tissue manufacturing facilities (see Fig. 1) in Canada, built as mentioned above thanks to major structuring investments by the Canadian Foundation for Innovation, provincial governments, hospital foundations, and others: the Center of Excellence in Cell Therapy (D.C. Roy, scientific director [SD]) at Hôpital Maisonneuve-Rosemont in Montréal, the Centre Multidisciplinaire de Développement du Génie Tissulaire (L. Germain, SD) at Université Laval in Québec City, the Orsino Cell Therapy Translational Research Laboratory at 
FIG. 1. CellCAN, a distributed model.

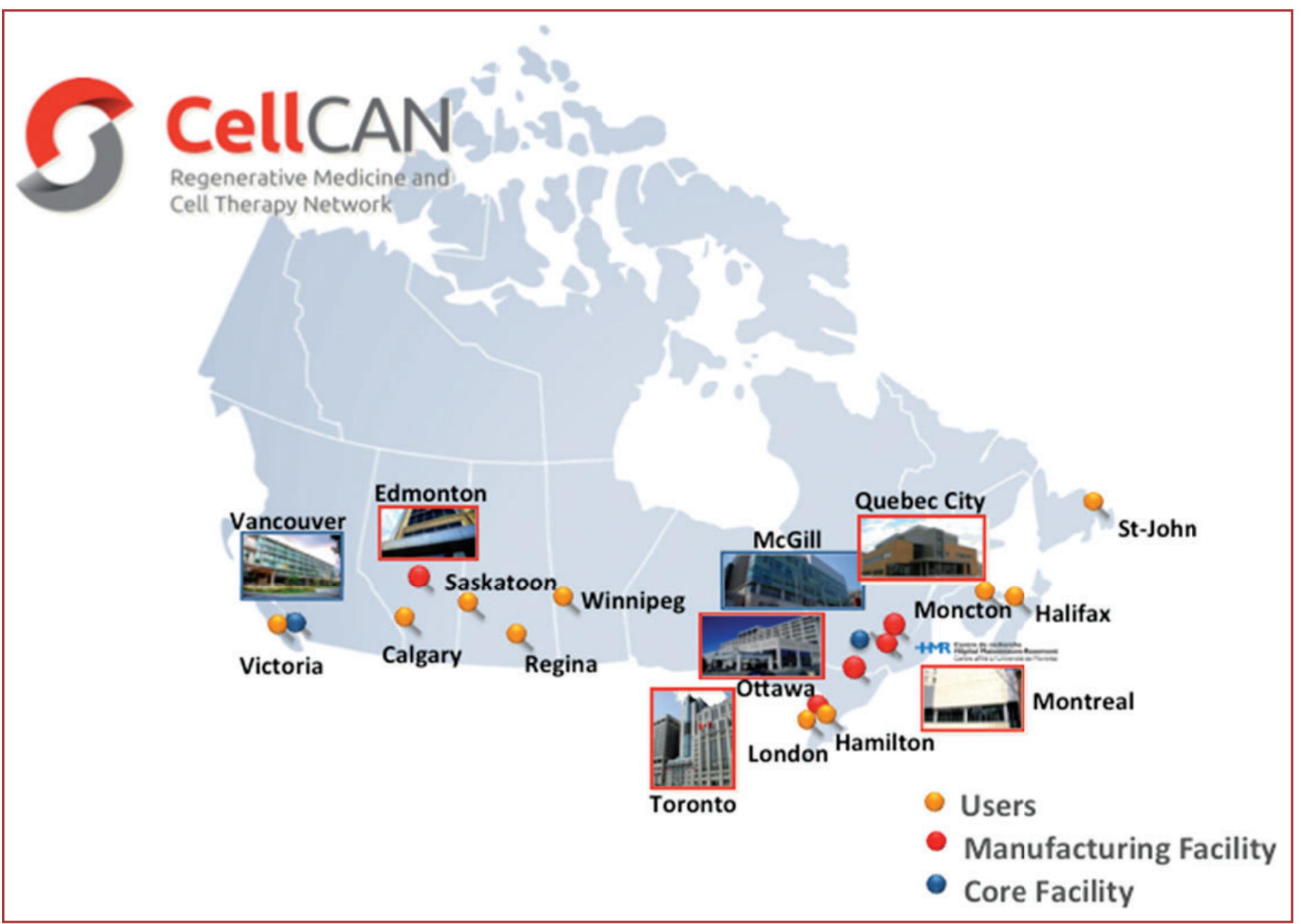

University Health Network (A. Keating, $\mathrm{SD}$ ) in Toronto, the Ottawa Hospital Research Institute (D. Stewart, SD) in Ottawa, and the Alberta Cell Therapy Manufacturing (G. Korbutt, SD) in Edmonton.

These facilities represent a combined capacity of over 4500 square meters with the ability to isolate, grow, and manipulate cells in a tightly controlled (temperature, humidity, pressure, particles, etc.) and highly regulated environment ensuring their safe and traceable administration to patients across Canada.

Two transversal, integrated, and synergistic cores further enrich CellCAN's cell and tissue manufacturing facilities ecosystem:
- The Centre for Genomics and Policy $(C G P)$, led by Bartha Knoppers, is a world-renowned hub for research into international law, ethics, and policy, at the crossroads of the legal, medical, and public policy fields. At the forefront of major national and international genomic health sciences initiatives, the CGP is devoted to developing best practices, as well as regulatory and ethical-policy guidance for RMCTs, among other fields.

- The UBC-Biotechnology Core is a transversal bioengineering core facility to all cell and tissue manufacturing within CellCAN. This group, led by Dr. James Piret, focuses on optimization of therapeutic cell bioprocesses through cellular physiology and engineering approaches.
This aims to improve the design and operation of stem cell and tissue manufacturing and ultimately establish robust and noninvasive culture process controls through analysis, prediction, and validation of process performance for clinical-grade production.

CellCAN aims to synergize and accelerate the operations of these cellhandling facilities, thus providing a panCanadian resource in RMCT. CellCAN provides (1) support and optimization of the operations in Canadian GMP cell manufacturing facilities by distributing efforts needed to develop new procedures and protocols; (2) expertise and versatile infrastructures for translation of research innovations into clinical cell therapy manipulations; (3) facilitation of 
national interoperability and harmonization of ethical, legal, and regulatory requirements; and (4) coordination and promotion of multicenter trials, and propels Canada as a particularly attractive destination for clinical trials in RMCT.

\section{CellCAN, a unique hub for the advancement of cell and tissue therapy}

CellCAN creates a national enterprise bringing together a community of researchers, industry, clinicians, funders, and regulators, and aligning it with cellhandling facilities involved in processing cell and other products to ensure capacity and know-how for stem cell research and efficient execution of cell therapy clinical trials in Canada.

This distributed model with multiple centers across major Canadian cities is better suited to support the development of cell and tissue therapy products, since they often require to be manufactured in close proximity to the clinical centers conducting early phase trials due to their limited shelf life. This model also builds on specific areas of expertise developed at each subsidiary, thus promoting

\section{FIG. 2. Main Canadian cell manufacturing centers and primary areas of expertise.}

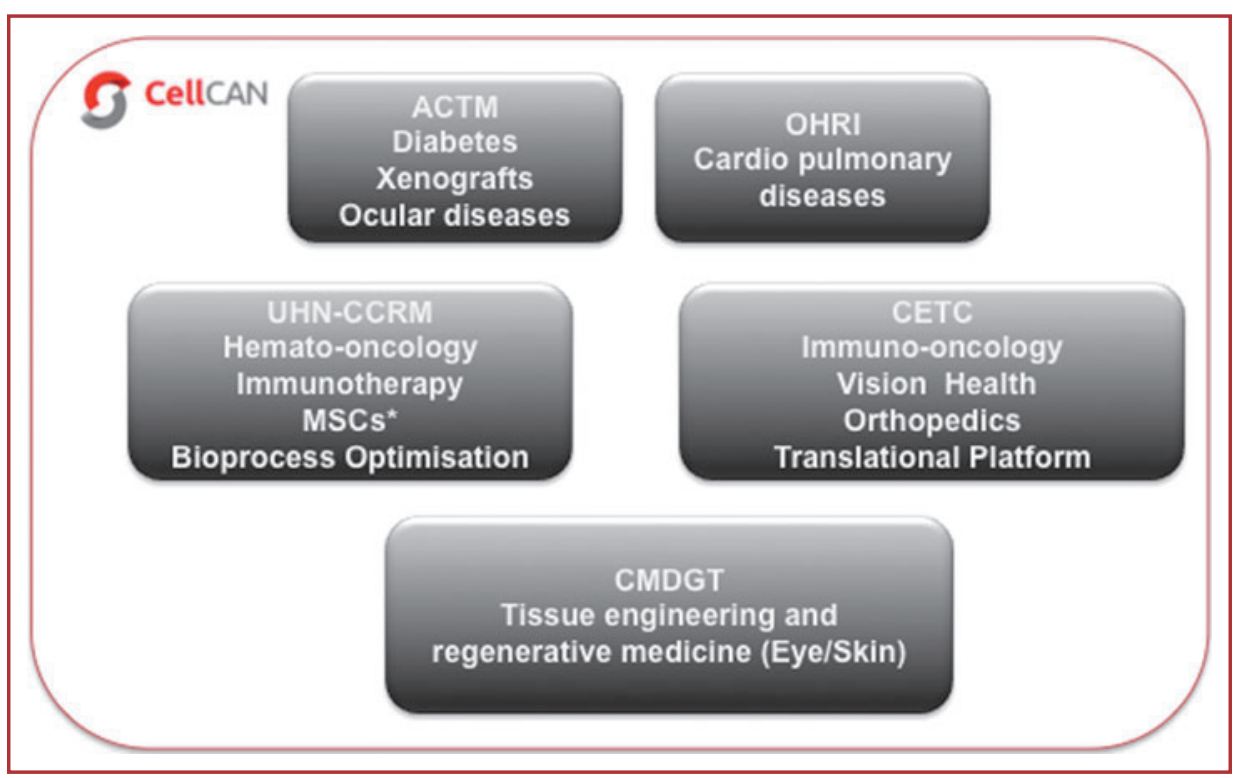

duplication of manufacturing efforts Providing such a focal point for cell and tissue therapy catapults the country to the forefront of the rapidly developing field cell therapy to Canadian patients. Figure 2 shows the main Canadian cell manufacturing centers and primary areas of expertise.

CellCAN's members have demonstrated their expertise and leadership in translating their scientific and technical knowledge into clinical breakthroughs. Indeed, critical unresolved medical challenges from pulmonary hypertension, hematopoietic stem cell transplantation, orphan disease like Fabry's disorder, osteoarthritis, hematological malignancy, diabetes to cornea transplantation are being tackled by the academic and private users of CellCAN's facilities. Furthermore, by allowing the major cell and tissue manufacturing facilities in Canada and their teams to coalesce and synergize their efforts with those of the Canadian National Transplant Research Program (CNTRP), CellCAN is transforming the organ transplant paradigm. CNTRP aims to make new scientific discoveries and develop healthcare practices, including cell therapy, to address barriers to cell, tissue, and organ donation so as to increase access and optimize performance of transplantation for Canadians in need. of RMCT, accelerating the delivery of

CellCAN is also expected to act as a beacon for industry-sponsored trials by accelerating regulatory approval and offering greater regulatory certainty. To that end, it will provide regulatory advice and build regulatory roadmaps and toolkits and clinical trial protocols to enable interoperability and harmonization between institutional/national/international jurisdictions and regulatory agencies (e.g., Health Canada, FDA, EMA). Its activities will generate significant regulatory advantages for Canada, providing a ready conduit for dialog between clinicians, manufacturers, and regulatory agencies on specific and broad regulatory concerns. In addition, it will provide standardized contracting protocols and facilitated patient accrual via the clinician networks associated with the network.

\section{Mobilizing knowledge}

Funding was recently awarded to CellCAN (CAD\$3 million, from the NCEs of Canada, Ronald and Herbert Black and Hôpital Maisonneuve-Rosemont Foundation, and several charities) to bridge knowledge gaps between cell manufacturers, and develop privileged links with scientists, clinicians, regulators, industry, and the end users, which include patients and the lay public.

CellCAN is therefore uniquely positioned to address issues surrounding RMCT knowledge mobilization. It will diffuse information, data, and knowledge to accelerate the implementation of novel cellular therapies that will benefit investigators - and patients - by providing instructions in areas ranging from translational development to the scale-up of a product that is intended for use in human clinical trials. CellCAN will reach out to investigators new to the field and provide them with recommended applications of the current GMP and good tissue practice guidelines. Collectively, this will lead to tremendous health (better treatment for patients), social (less emotional stress for family), and economical (creation of jobs) 
benefits for Canada. Through this unique collaborative approach with not-for-profit organizations, the knowledge capacity and access of patients to cutting-edge care will be enhanced tremendously.

\section{CellCAN: shaping the} future of RMCT in Canada

CellCAN is uniquely positioned to accelerate the implementation of RMCT

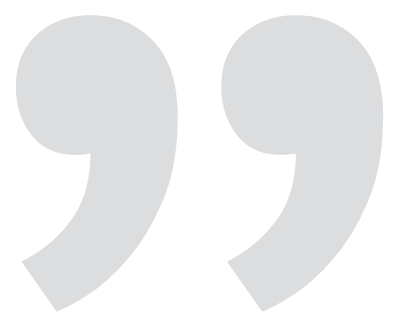

The CellCAN model could serve as a useful benchmark to accelerate regenerative medicine and cell therapy implementation in other countries. in Canada and disseminate novel developments and findings, thus significantly contributing to the world's knowledge in cellular therapeutics. As such, the CellCAN model could also serve as a useful benchmark to accelerate RMCT implementation in other countries.

\section{Author Disclosure}

\section{Statement}

No competing financial interests exist.

$$
\begin{aligned}
& \text { Web Resources } \\
& \text { - CellCAN-www.cellcan.com } \\
& \text { - Canadian National Transplant } \\
& \text { Research Program-www.cntrp.ca } \\
& \text { - The Stem Cell Network-www } \\
& \text {.stemcellnetwork.ca/ } \\
& \text { - Centre for Commercialization of } \\
& \text { Regenerative Medicine- } \\
& \text { http://ccrm.ca/ }
\end{aligned}
$$

\section{REFERENCES}

1. Heidenreich PA, JG Trogdon, OA Khavjou, et al. (2011). Forecasting the future of cardiovascular disease in the United States: a policy statement from the American Heart Association. Circulation 123:933-944.

2. Mason C and P Dunnill (2008). The strong financial case for regenerative medicine and the regen industry. Regen Med 3:351-363.

3. Mason C and E Manzotti. (2010). Regenerative medicine cell therapies: numbers of units manufactured and patients treated between 1988 and 2010. Regen Med 5:307-313.

4. Brindley DA, NL Davie, WA Sahlman, et al. (2012). Promising growth and investment in the cell therapy industry during the first quarter of 2012. Cell Stem Cell 10:492-496.

5. Mason C, J Mason, EJ Culme-Seymour, GA Bonfiglio and BC Reeve. (2013). Cell therapy companies make strong progress from October 2012 to March 2013 amid mixed stock market sentiment. Cell Stem Cell 12:644-647.

6. Mason C, MJ McCall, EJ CulmeSeymour, et al. (2012). The global cell therapy industry continues to rise during the second and third quarters of 2012. Cell Stem Cell 11:735-739.

7. EUR 22836 (2007). Improving Knowledge Transfer Between Research Institutions and Industry Across Europe: Embracing Open Innovation. Office for Official Publications of the European Communities, Luxembourg, pp 1-36. 